\title{
Sclera to the Rescue in the Covid-19 Pandemic- Therapeutic Scleral Grafting in Perforated Corneal Ulcers due to Donor Cornea Shortage
}

\section{Radhika Natarajan*, Hiren D Matai and Niveditha Narayanan}

Department of Cornea and Refractive Surgery, Sankara Nethralaya, Medical Research Foundation, India

*Corresponding author: Natarajan R, Old No. 18, New No. 41, College Road, Nungambakkam, Department of Cornea and Refractive Surgery, Sankara Nethralaya, Medical Research Foundation, Chennai, Tamil Nadu - 600 006, India, Tel: +91-98403 08181; E-mail: 100radsam@gmail.com

Received: February 08, 2021; Accepted: February 18, 2021; Published: February 25, 2021

\begin{abstract}
PURPOSE: We report two cases of perforated fungal corneal ulcers, who successfully underwent large therapeutic scleral grafts as globe salvaging procedures, due to the dearth of donor corneal tissue in the COVID-19 pandemic.

METHODS: Both patients underwent therapeutic grafting for large corneal perforations with trephined donor scleral grafts. Suturing was done using 9-0 nylon suture. One scleral graft was covered with a conjunctival flap and the other with a total tarsorrhaphy. Post-operatively, topical antifungal medications were continued.

RESULTS: At the one-month follow-up, both the scleral grafts were found to be healing well. There was no recurrence of infection. Anatomical integrity was restored in both the eyes.

CONCLUSION: Therapeutic scleral grafting can be done for large corneal perforations, in case of absolute non-availability of donor corneal tissue. Certain steps like providing access to vascularisation of the graft, need specific attention during the procedure. These scleral grafts can provide tectonic stability in perforated corneal ulcers.
\end{abstract}

\section{Introduction}

Infectious keratitis with large perforations requires therapeutic penetrating keratoplasty (TPK) to eliminate infection and restore the tectonic integrity of the globe [1,2]. Donor corneal tissue of fair to good quality is usually used for this surgery for good anatomical outcome [3]. 
www.yumedtext.com | February-2021 | ISSN: 2582-5038 | https://dx.doi.org/10.46527/2582-5038.178

The ongoing SARS-CoV-2 (COVID-19) pandemic has imposed unprecedented re-strictions in eye banking across the world resulting in extreme donor corneal tissue shortage or non-availability, due to which long term corneal preservation methods are being galvanized into action [4]. This too is entirely not enough to meet emergency corneal needs.

We hereby report two cases of perforated corneal ulcers, who successfully underwent large therapeutic scleral grafts as globe salvaging procedures, due to the dearth of donor corneal tissue in the COVID-19 pandemic.

\section{Case Report}

A 35-year-old man, suffering from Xeroderma Pigmentosum, presented with recurrent fungal keratitis in the therapeutic corneal graft in his only seeing eye. His right eye had been eviscerated for corneal infection, in childhood. He had undergone two penetrating keratoplasties and a cataract surgery with intraocular lens implantation in the left eye some years back, elsewhere. Under our care, he had undergone lower lid excision biopsy for basal cell carcinoma and later TPK for fungal keratitis. The TPK graft remained stable for two weeks, after which he was lost to follow up due to the COVID-19 pandemic and the ensuing lockdown.

He reported back with a sudden reduction of vision in the left eye of two weeks duration and a 5 mm central graft infiltrate involving the deep stroma, 30\% stromal thinning, and an overlying epithelial defect. A posterior chamber intraocular lens (PCIOL) could be made out hazily. B-scan ultrasonography (USG) was unremarkable. Corneal scraping revealed septate fungal filaments.

The thinning and infiltrate progressed despite optimal medical therapy for which the patient underwent cyanoacrylate glue application with bandage contact lens (BCL) for tec-tonic stability. A week later, he presented with loosening of the glue and an underlying corneal perforation. Since the surrounding corneal graft was unhealthy, it was decided against repeating the cyanoacrylate glue or attempting any patch graft. A full-thickness therapeutic scleral graft was planned due to the dearth of donor cornea and difficulty in the procurement of long-term preserved corneal tissue.

Under peribulbar anesthesia, the glue and BCL were removed (FIG. 1A). The necrotic corneal graft was excised. The anterior chamber (AC) was thoroughly irrigated. Anterior synechiae were released. The iris appeared healthy and the PCIOL was well in place (FIG. 1B). A good red fundal glow was visible. Two peripheral iridectomies were done. A 9 mm scleral graft was punched out of a previously excised larger disc of glycerol pre-served donor sclera. This was then cleaned well of uveal pigment and washed thoroughly before suturing it to the host corneal rim with 16 interrupted 9-0 nylon sutures. The AC was formed after washing out the viscoelastic and the wound checked for leakage. The knots were trimmed and left unburied on the graft side to avoid cheese wiring due to tissues of unequal strength (FIG. 1C). The graft was covered with an amniotic membrane sutured beyond the limbus to reduce symptoms due to the knots and facilitate healing. The conjunctiva was boggy and bleeding to touch and could not be satisfactorily advanced over the scleral graft. The earlier excision biopsy of the lower lid was also causing mild incomplete lid closure. So, a complete tarsorrhaphy was done to close the eye for healing (FIG. 1D). A sliver of opening nasally allowed for some visibility for examination and instilling drops. 


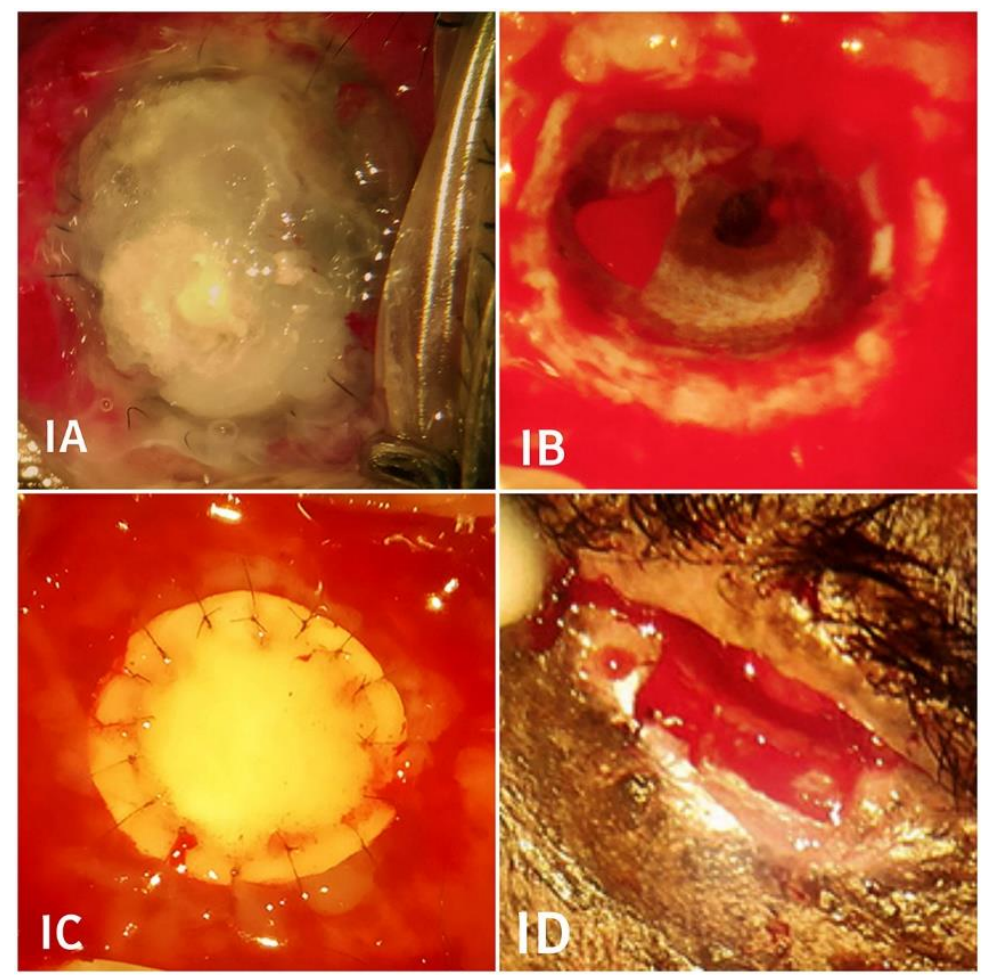

FIG. 1A. Case 1 Showing Recurrent Infection with Loosened Cyanoacrylate Glue. FIG. 1B. Healthy Iris Tissue after Anterior Chamber Irrigation. FIG. 1C. Large Therapeutic Scleral Graft Sutured in Place. FIG. 1D. Eye Closed with Tarsorrhaphy.

Post-operatively, he was continued on topical antifungal drops. The excised host button did not show any growth. At two weeks, the scleral graft was healing well with the tarsorrhaphy in place with a slit opening in the interpalpebral area. Digital tonometry and USG of the posterior segment were normal. He needed to be under the care of his oncologist for pulmonary metastases. At the one-month review, the scleral graft and overlying tarsorrhaphy were found to be effective in maintaining the tectonic stability of the eye, during his extended oncological treatments.

Another patient, a 40-year-old lady, presented with a perforated corneal ulcer with aphakia and vitreous prolapse in the right eye, after being on treatment for fungal keratitis for 2 weeks. She too underwent therapeutic scleral patch grafting due to the non-availability of donor cornea. The initial surgical steps were similar to the previous case.

The ovoid-shaped perforation measured $8 \mathrm{~mm} \times 9 \mathrm{~mm}$. A limited anterior vitrectomy was done. A $10 \mathrm{~mm}$ scleral graft was trephined. An end-to-end approximation was difficult in view of cheese wiring. The scleral graft was sutured with 16 interrupted 9-0 nylon sutures. Intra-cameral Voriconazole injection was given. A total conjunctival flap was fashioned by performing a 360-degree peritomy. The flap edges were mobilized superiorly and inferiorly to cover the scleral graft and approximated with a running purse-string suture. The eye was washed with a 5\% Povidone Iodine solution and patched.

The host corneal button remnant sent for microbiology showed Aspergillus flavus. The patient was continued on topical antifungal drops postoperatively. On the first post-operative day, USG showed a shallow choroidal detachment in the right eye 
which resolved in a week. The scleral graft started to show vascularisation in the second post-operative week (FIG. 2) and was stable at the one-month follow-up.

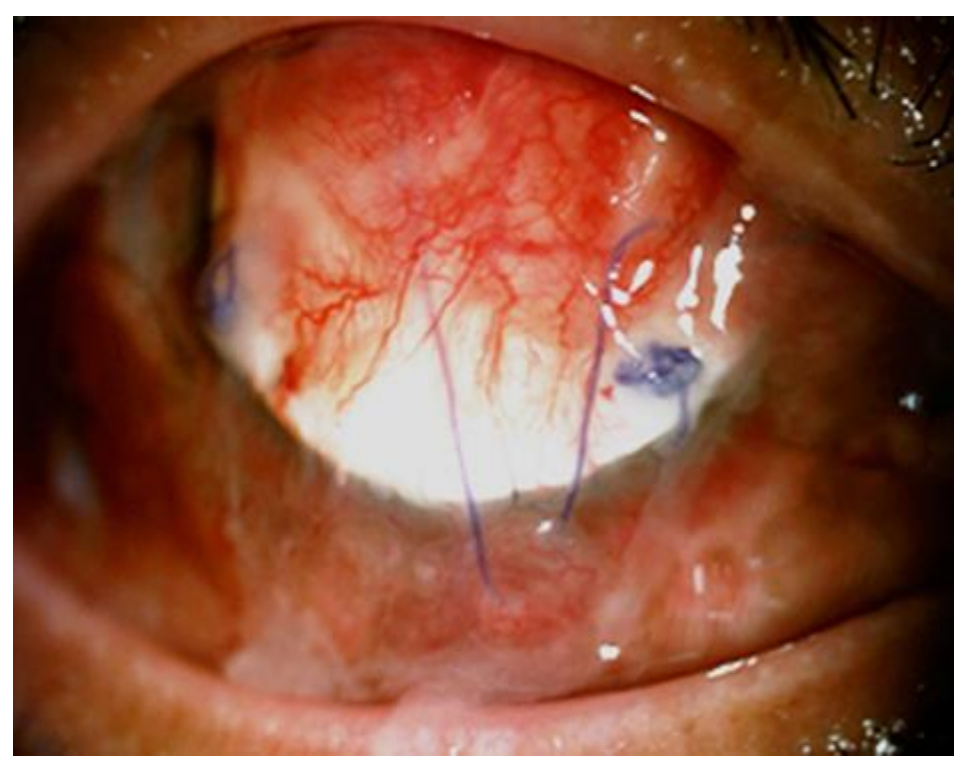

FIG. 2. Post-Operative Picture of Case 2 showing Scleral Graft with Overlying Conjunctival Flap.

\section{Discussion}

Perforated corneal ulcers constitute an ophthalmic emergency that mandates urgent tec-tonic repair. Otherwise, expulsion of ocular contents and intraocular spread of infection can result in significant ocular morbidity. Corneal perforations up to $2 \mathrm{~mm}$ to $3 \mathrm{~mm}$ in diameter can be sealed with Cyanoacrylate glue if the surrounding tissue is healthy. Alternatively, a Tenon's patch graft or a multi-layered amniotic membrane graft can also be used [5].

However, large corneal perforations with surrounding unhealthy tissue need TPK.1 TPK is done to get rid of the infection and impart tectonic stability to the globe. Fair to good donor corneal tissue is used to achieve a good anatomical outcome.

The COVID-19 pandemic has brought about unprecedented restrictions in donor cornea retrieval causing a downtrend in the global elective keratoplasty rates between March and July 2020. Eye banks have resorted to putting available tissue into long term storage to be used for emergency situations. Glycerol and cryopreservation are the common methods being adopted around the world for long term corneal preservation $[4,6]$.

In case of absolute non-availability of corneal tissue, other surgical measures for corneal perforation in dire contingency include a Gundersen\&rsquo's conjunctival flap or a total tarsorrhaphy [7,8]. Though scleral patch grafts are done for various indications, its use in corneal perforation repair is anecdotal [9]. A partial thickness scleral flap from the patient\&rsquo;s own eye has also been described in small peripheral perforations [10]. We decided to go ahead with therapeutic scleral grafting when two of our patients needed urgent repair of large corneal perforations, at a time of total non-availability of donor corneal tissue due to the COVID-19 pandemic. 
www.yumedtext.com | February-2021 | ISSN: 2582-5038 | https://dx.doi.org/10.46527/2582-5038.178

During the scleral grafting procedure, some specific steps were performed. These included trephining the final graft from a manually cut large disc of sclera, removal of iris tissue from the sclera, thorough cleaning and irrigating the scleral graft, not attempting to thin out the donor sclera before grafting as a full-thickness closure was needed, tapering of the graft edge to slope for better approximation, leaving the knots exposed but trimmed to prevent cheese wiring due to burying and covering the graft with a conjunctival flap to promote vascularisation. Scleral grafts need to vascularise without which necrosis and infection can occur [9]. As the conjunctiva was boggy, friable, and bleeding to touch, a near-total tarsorrhaphy was done in the other patient. Both the patients were found to be healing well post-operatively, at the one-month follow-up. The possibility of optical penetrating keratoplasty with its risks and benefits, once the eyes quieten completely and donor tissues become available, was explained to them.

In summary, therapeutic scleral grafting can be done for large corneal perforations, in case of absolute non-availability of donor corneal tissue. Certain steps, like providing access to vascularisation of the graft, need specific attention during the procedure. Such scleral grafts can provide tectonic integrity in perforated corneal ulcers.

\section{REFERENCES}

1. Sharma N, Sachdev R, Jhanji V, et al. Therapeutic keratoplasty for microbial keratitis. Curr Opin Ophthalmol. 2010;21(4):293-300.

2. Raj A, Bahadur H, Dhasmana R. Outcome of therapeutic penetrating keratoplasty in advanced infectious keratitis. J Curr Ophthalmol. 2018;30(4):315-20.

3. Rao GN, Garg P, Sridhar MS. Therapeutic penetrating keratoplasty. In: Brightbill FS, editor. Corneal surgery: theory, technique and tissue. St. Louis: Mosby, USA; 2009. 439-44 p.

4. Chaurasia S, Das S, Roy A. A review of long-term corneal preservation techniques: Relevance and renewed interests in the COVID-19 era. Indian J Ophthalmol. 2020;68(7):1357-63.

5. Deshmukh R, Stevenson LJ, Vajpayee R. Management of corneal perforations: An update. Indian J Ophthalmol. 2020;68(1):7-14.

6. Chaurasia S, Sharma N, Das S. COVID-19 and eye banking. Indian J Ophthalmol. 2020;68(6):1215-6.

7. Portnoy SL, Insler MS, Kaufman HE. Surgical Management of Corneal Ulceration and Perforation. Surv Ophthalmol. 1989; 34(1):47-58.

8. Pakarinen M, Tervo T, Tarkkanen A. Tarsorraphy in the Treatment of Persistent Corneal Lesions. Acta Ophthalmol Suppl. 1987;182:69-73.

9. Wang SY, Tian Y, Zhu HF, et al. Allogeneic sclera graft combined autologous conjunctival flap for repairing the emergent corneal perforation. Ann Eye Sci. 2017;2(10):66.

10. Larsson S. Treatment of perforated corneal ulcer by autoplastic scleral transplantation. Br J Ophthalmol. 1948;32(1):54-7. 